\title{
Instrumentos para valorar al paciente con insuficiencia cardíaca avanzada: una revisión de la literatura
}

\author{
Assessment instruments for patients with advanced heart failure: \\ a review of the literature
}

\section{J. Martín', A. Carvajal ${ }^{1,2,4}$, M. Arantzamendi ${ }^{1,3,4}$}

\section{RESUMEN}

Una cuarta parte de las personas con insuficiencia cardíaca se encuentran en una etapa avanzada de la enfermedad durante la cual experimentan numerosos síntomas, comunes y angustiantes, que impactan en todas las esferas de la persona. En este contexto, la valoración y el seguimiento clínico de los pacientes se debe realizar de manera frecuente. A través de esta revisión, se pretende identificar cuáles son los instrumentos que se utilizan en la valoración de enfermos con insuficiencia cardíaca avanzada. Para este trabajo se ha considerado instrumento a cualquier tipo de cuestionario, escala, test o prueba funcional, que se utilice para valorar algún aspecto de estos enfermos. Se han identificado 49 herramientas que permiten valorar síntomas, aspectos psicológicos, cognitivos, espirituales y de calidad de vida. La información proporcionada sobre los instrumentos más utilizados y su disponibilidad y aplicabilidad constituye un primer paso para su posible integración en la práctica clínica diaria. De este modo, los profesionales que trabajan con estos enfermos, podrán mejorar la identificación de necesidades específicas, posibilitando el posterior manejo de las mismas y su seguimiento.

Palabras clave. Insuficiencia Cardíaca. Valoración. Cuestionario. Escala.

\begin{abstract}
A quarter of the people with heart failure are at an advanced stage of the disease, during which they experience numerous common and distressing symptoms that have an impact on all spheres of their life. In this context, there is a need for frequent assessment and clinical monitoring of patients. The aim of this review is to identify the instruments used in assessing patients with advanced heart failure. For the purposes of this study, any type of questionnaire, scale or functional test used to assess some aspect of these patients was considered to be an instrument. Forty-nine tools were identified that make it possible to assess symptoms, psychological, cognitive and spiritual aspects and quality of life. The information provided on the most used instruments and their availability and applicability is a first step for their possible integration into daily clinical practice. Thus, professionals who work with these patients can improve the identification of specific needs, enabling their subsequent management and monitoring.
\end{abstract}

Keywords. Heart failure. Assessment. Questionnaire. Scale.
1. Facultad de Enfermería, Universidad de Navarra.

2. Clínica Universidad de Navarra.

3. Programa ATLANTES. Instituto Cultura y Sociedad. Proyecto Atlantes. Universidad de Navarra.

4. Instituto de Investigación Sanitaria de Navarra (IdiSNA).

Recepción: 23 de febrero de 2015

Aceptación provisional: 1 de junio de 2015

Aceptación definitiva: 29 de junio de 2015

\section{Correspondencia:}

Jesús Martín

Facultad de Enfermería

Universidad de Navarra

Campus Universitario

31008 Pamplona (Navarra)

E-mail: jmartin.19@alumni.unav.es 


\section{INTRODUCCIÓN}

Las enfermedades cardiovasculares en España, al igual que en el resto de los países occidentales, constituyen la primera causa de muerte en el conjunto de la población ${ }^{1}$. Dentro del grupo de enfermedades que las conforman se encuentra la insuficiencia cardíaca (IC). Se trata de una enfermedad común, incapacitante y potencialmente mortal que, además, constituye la principal causa de hospitalización en personas mayores de 65 años ${ }^{2}$.

En el mundo desarrollado, esta enfermedad afecta aproximadamente al $2 \%$ de la población adulta, con una prevalencia que aumenta de forma exponencial con la edad $^{2}$. La prevalencia en España fue del $4,7 \%^{3}$ con una incidencia en la población de $3,90 / 1000$ personas-año en el $2007^{2}$. Así mismo, en cuanto a la mortalidad, conforme a los últimos datos del Instituto Nacional de Estadística, durante el 2013 la IC se cobró la vida de 43.256 personas (27.540 mujeres y 15.716 hombres) en nuestro medio, posicionándose como tercera causa de muerte entre las enfermedades cardiovasculares, detrás de las cerebrovasculares y otras enfermedades del corazón ${ }^{4}$. Por otra parte, la IC constituye una enfermedad, que en gran parte de los casos, progresa hacia una insuficiencia cardíaca avanzada (ICA) ${ }^{5}$. Ésta se define como un estado en el que el paciente presenta una severa disfunción cardíaca con marcada disnea, fatiga o síntomas relacionados a hipoperfusión tisular en reposo o con mínimos esfuerzos, a pesar de máxima terapia. Esta conceptualización concuerda con el estadio D propuesto por la American College of Cardiology/American Heart Association ${ }^{6}$. Se estima que casi un cuarto de los pacientes con IC progresará a una ICA ${ }^{7}$. Además, aproximadamente el $10 \%$ de las personas con IC se encuentran en etapa terminal de la enfermedad ${ }^{8}$. Por todo lo comentado anteriormente, la ICA constituye actualmente un trastorno progresivo y letal, cuya prevalencia, incidencia y mortalidad siguen en ascenso ${ }^{5,9}$. Esto la convierte en uno de los problemas asistenciales de mayor magnitud en los países desarrollados por la elevada y progresiva morbimortalidad que conlleva y el elevado coste humano, técnico y económico derivado de su atención ${ }^{10}$.

A su vez, esta enfermedad está caracterizada por una mala respuesta al tratamiento farmacológico, los múltiples ingresos hospitalarios y los numerosos síntomas que incapacitan a la persona ${ }^{5}$. La literatura muestra cómo estos síntomas, comunes y angustiantes, impactan en todas las esferas del paciente con ICA (física, psicológica, social y espiritual). De hecho, estos enfermos presentan un mayor número de síntomas físicos, una mayor tendencia a la depresión y un menor bienestar espiritual que los enfermos de cáncer durante los últimos 6 meses de vida ${ }^{11}$. Además, el sufrimiento provocado por dichos síntomas aumenta en los momentos cercanos al final de la vida ${ }^{12}$. Igualmente, se ha demostrado que los pacientes con ICA presentan grandes cambios de su estado de enfermedad a corto plazo, en comparación con otros pacientes en fases menos avanzadas de la misma $^{13}$. Por ello, la valoración y el seguimiento clínico de estos enfermos se debe realizar de manera frecuente, por lo que resulta útil conocer las herramientas que se disponen para la valoración de las dimensiones de la persona con ICA. El conocimiento de los distintos instrumentos que se disponen en la actualidad y su aplicación en la práctica clínica diaria para el cuidado de personas con ICA, puede ayudar a mejorar la identificación de necesidades específicas, el control de síntomas, reales o potenciales, y el manejo de las complicaciones de estos pacientes por parte de los profesionales de la salud.

Por ello, el objetivo principal de esta revisión es conocer los instrumentos que se utilizan para valorar a la persona con ICA.

\section{METODOLOGÍA}

Se llevó a cabo una búsqueda bibliográfica en las siguientes bases de datos: PubMed, CINHAL, Psycinfo, Cochrane Library y ScienceDirect. En la estrategia de búsqueda se emplearon las palabras clave "Instrumentos" y "Pacientes con Insuficiencia Cardíaca Avanzada” y sus sinónimos tradu- 
cidos al inglés, identificados de los términos MeSH, del Tesauro y de la literatura. Se utilizaron operadores booleanos ("AND" y "OR") y el truncamiento de palabras para incluir posibles variaciones utilizadas en la literatura, tal y como muestra la figura 1.
Además, para la selección de artículos se establecieron los criterios de inclusión y exclusión reflejados en la tabla 1. Finalmente, se limitó la búsqueda a los últimos 10 años, al idioma (inglés o español) y a la población adulta (mayores de 18 años).

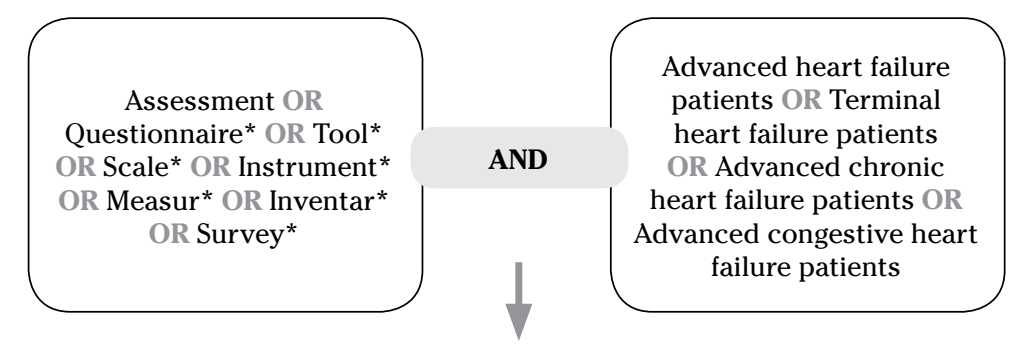

\begin{tabular}{|c|c|c|c|c|}
\hline \multicolumn{5}{|c|}{ Primer paso: Búsqueda en las bases de datos } \\
\hline PubMed & CINHAL & Psycinfo & Cochrane & ScienceDirect \\
\hline 500 & 147 & 19 & 63 & 116 \\
\hline \multicolumn{5}{|c|}{ Segundo paso: Selección de publicaciones según criterios de inclusión y exclusión } \\
\hline PubMed & CINHAL & Psycinfo & Cochrane & ScienceDirect \\
\hline 9 & 6 & 3 & 2 & 5 \\
\hline \multicolumn{5}{|c|}{ Tercer paso: búsqueda utilizando la técnica de bola de nieve. } \\
\hline \multicolumn{5}{|c|}{ Búsqueda manual: 1} \\
\hline \multicolumn{5}{|c|}{ Cuarto paso: Artículos finalmente seleccionados } \\
\hline \multicolumn{5}{|c|}{25 de las bases de datos y 1 de la técnica bola de nieve } \\
\hline
\end{tabular}

Figura 1. Estrategia y resultados de la búsqueda bibliográfica

Tabla 1 Criterios de selección de artículos

\section{Criterios de inclusión}

Estudios que utilicen instrumentos que valoren al paciente con ICA.

Estudios cuantitativos, cualitativos y revisiones de la literatura.

\section{Criterios de exclusión}

Estudios que utilicen instrumentos que valoren a pacientes con otras patologías diferentes a la ICA.

Estudios que utilicen instrumentos que valoren a los familiares.

Estudios que utilicen instrumentos que valoren a la población pediátrica. 
De la estrategia de búsqueda se obtuvieron un total de 845 artículos. Uno de los investigadores realizó un cribado seleccionando los artículos por título y resumen. Posteriormente, se realizó una lectura crítica de los artículos seleccionados aplicando los criterios de selección y eliminando los duplicados. Este proceso se llevó a cabo por dos investigadores. Finalmente, se in- cluyeron 25 artículos para el análisis de los resultados. Así mismo, a través de una búsqueda manual en las listas de referencias de los artículos seleccionados (técnica de bola de nieve) se añadió un estudio relevante (Fig. 1). Por consiguiente, se seleccionaron un total de 26 artículos ${ }^{12,14-38}$ que quedan resumidos en la tabla 2 .

Tabla 2. Resumen artículos búsqueda bibliográfica.

\begin{tabular}{|c|c|c|c|c|c|}
\hline Autores & Año & País & Objetivo & Población & Instrumentos \\
\hline $\begin{array}{l}\text { Evangelista, } \\
\text { LS y col }{ }^{14}\end{array}$ & 2014 & EEUU & $\begin{array}{l}\text { Describir los niveles de carga de } \\
\text { síntomas en la primera consulta } \\
\text { de cuidados paliativos y tres } \\
\text { meses después. }\end{array}$ & $\begin{array}{l}\mathrm{N}=36 \\
\text { pacientes } \\
\text { con IC } \\
\text { clase II-III. }\end{array}$ & - Edmonton Symptom Assessment Scale (ESAS) \\
\hline $\begin{array}{l}\text { Park, CL } \\
\mathrm{y} \mathrm{col}^{15}\end{array}$ & 2014 & EEUU & $\begin{array}{l}\text { Examinar la relación entre } \\
\text { múltiples dimensiones de } \\
\text { espiritualidad y de bienestar. }\end{array}$ & $\begin{array}{l}\mathrm{N}=111 \\
\text { pacientes } \\
\text { con ICA. }\end{array}$ & $\begin{array}{l}\text { - Minnesota Living with Heart Failure Questionnaire } \\
\text { (MLHFQ) } \\
\text { - SF-12 Health Survey. } \\
\text { - CES-D scale. } \\
\text { - Satisfaction with Life Scale (SWLS) } \\
\text { - Religious Strain Scale } \\
\text { - Brief Multidimensional Measure of Religion/ } \\
\text { Spirituality (BMMR/S) }\end{array}$ \\
\hline $\begin{array}{l}\text { Bunyamin, V } \\
\mathrm{y} \mathrm{col}^{16}\end{array}$ & 2013 & Alemania & $\begin{array}{l}\text { Examinar la variabilidad de la } \\
\text { calidad de vida en pacientes con } \\
\text { ICA. }\end{array}$ & $\begin{array}{l}\mathrm{N}=318 \\
\text { pacientes } \\
\text { con ICA. }\end{array}$ & $\begin{array}{l}\text { - Heart Failure Survival Score (HFSS) } \\
\text { - Minnesota Living with Heart Failure Questionnaire } \\
\text { (MLHFQ) } \\
\text { - Hospital Anxiety and Depression Scale (HADS) } \\
\text { - Mainz Coping Inventory (MCI) } \\
\text { - Community Healthy Activities Model Program for } \\
\text { Seniors (CHAMPS) }\end{array}$ \\
\hline $\begin{array}{l}\text { Aggarwal, A } \\
\mathrm{y} \mathrm{col}^{17}\end{array}$ & 2013 & EEUU & $\begin{array}{l}\text { Analizar la incidencia de la } \\
\text { desnutrición y evaluar su } \\
\text { importancia pronóstica en } \\
\text { pacientes con ICA. }\end{array}$ & $\begin{array}{l}\mathrm{N}=154 \\
\text { pacientes } \\
\text { con ICA. }\end{array}$ & - Mini Nutritional Assessment (MNA) \\
\hline $\begin{array}{l}\text { Lennie, TA } \\
\mathrm{y} \mathrm{col}^{18}\end{array}$ & 2013 & EEUU & $\begin{array}{l}\text { Testar la reducción del sodio en } \\
\text { la dieta con la administración de } \\
\text { suplementos de ácidos grasos } \\
\text { omega-3 en los síntomas de la IC, } \\
\text { la calidad de vida y el tiempo de } \\
\text { rehospitalización. }\end{array}$ & $\begin{array}{l}\mathrm{N}=175 \\
\text { pacientes } \\
\text { con ICA }\end{array}$ & $\begin{array}{l}\text { - Mini-cog } \\
\text { - Beck Depression Inventory II (BDI-II) } \\
\text { - Charlson Comorbidity Index (CCI) } \\
\text { - Memorial Symptom Assessment Scale Heart } \\
\text { Failure (MSAS-HF) } \\
\text { - Minnesota Living with Heart Failure Questionnaire } \\
\text { (LHFQ) }\end{array}$ \\
\hline $\begin{array}{l}\text { Goodlin, SJ } \\
\text { y coll }^{19}\end{array}$ & 2012 & EEUU & $\begin{array}{l}\text { Caracterizar el dolor en pacientes } \\
\text { con ICA. }\end{array}$ & $\begin{array}{l}\mathrm{N}=347 \\
\text { pacientes } \\
\text { con ICA }\end{array}$ & $\begin{array}{l}\text { - The Memorial Symptom Assessment Scale - } \\
\text { HF(MSAS-HF) } \\
\text { - The short form of the McGill Pain Questionnaire } \\
\text { (SF-MPQ) } \\
\text { - The Brief Pain Inventory (BPI) } \\
\text { - Kansas City Cardiomyopathy Questionnaire } \\
\text { (KCCQ) } \\
\text { - The Charlson Comorbidity Index }\end{array}$ \\
\hline $\begin{array}{l}\text { Strada, EA } \\
\mathrm{y} \mathrm{col}^{20}\end{array}$ & 2013 & EEUU & $\begin{array}{l}\text { Evaluar los niveles de bienestar } \\
\text { espiritual en poblaciones con } \\
\text { ICA o enfermedad pulmonar } \\
\text { obstructiva crónica }\end{array}$ & $\begin{array}{l}\mathrm{N}=206 \\
\text { pacientes } \\
\text { (103 con } \\
\text { ICA y } 103 \\
\text { con EPOC) }\end{array}$ & $\begin{array}{l}\text { - Functional Assessment of Chronic Illness Therapy- } \\
\text { Spiritual Well-Being (FACIT-Sp) Questionnaire. } \\
\text { - Multidimensional Index of Life Quality (MILQ) } \\
\text { - The Memorial Symptom Assessment Scale (MSAS) } \\
\text { - The Rand Mental Health Inventory (MHI) } \\
\text { - The Sickness Impact Profile (SIP) }\end{array}$ \\
\hline
\end{tabular}




\begin{tabular}{|c|c|c|c|}
\hline Autores & Año & País & Objetivo \\
\hline $\begin{array}{l}\text { Buck, HG } \\
\mathrm{y} \mathrm{col}^{21}\end{array}$ & 2012 & EEUU & $\begin{array}{l}\text { Describir la relación entre } \\
\text { auto-cuidado y calidad de vida } \\
\text { en mayores de } 65 \text { años con IC } \\
\text { moderada-avanzada. }\end{array}$ \\
\hline $\begin{array}{l}\text { Gaviria, M } \\
\mathrm{y} \mathrm{col}^{24}\end{array}$ & 2011 & EEUU & $\begin{array}{l}\text { Destacar la importancia de la } \\
\text { valoración del deterioro cognitivo } \\
\text { en pacientes con ICA }\end{array}$ \\
\hline $\begin{array}{l}\text { Hallas, CN } \\
\mathrm{y} \mathrm{col}^{25}\end{array}$ & 2011 & UK & $\begin{array}{l}\text { Identificar las variables } \\
\text { psicológicas y clínicas que } \\
\text { predicen el estado de ánimo y } \\
\text { calidad de vida para las personas } \\
\text { con diagnóstico de ICA. }\end{array}$ \\
\hline
\end{tabular}

\begin{tabular}{|c|c|c|c|}
\hline $\begin{array}{l}\text { Lee, CS } \\
\mathrm{y} \mathrm{col}^{26}\end{array}$ & 2011 & EEUU & $\begin{array}{l}\text { Comprobar si múltiples síntomas } \\
\text { podrían ser identificados y } \\
\text { asociados con diferencias en la } \\
\text { calidad de vida }\end{array}$ \\
\hline
\end{tabular}

Población Instrumentos

$\mathrm{N}=207 \quad$ - Self-care of Heart Failure Index

pacientes - Minnesota Living with Heart Failure

con IC Questionnaire (MLHFQ)

moderada -

avanzada

Revisión - Mini-Mental State Examination (MMSE)

- The Montreal Cognitive Assessment
$\mathrm{N}=146$ - Illness Perception Questionnaire-Revised (IPQ-R)
pacientes - World Health Organization Quality of Life
con ICA Measure (WHOQOL)
- Hospital Anxiety and Depression Scale (HADS)
- Minnesota Living with Heart Failure Questionnaire (MLHFQ)

$\begin{array}{ll}\mathrm{N}=74 & \text { - Heart Failure Somatic Perception Scale (HFSPS) } \\ \text { pacientes } & \text { - Brief Symptom Inventory (BSI) } \\ \text { con ICA } & \text { - Epworth Sleepiness Scale (ESS) } \\ & \text { - Minnesota Living with Heart Failure } \\ & \text { Questionnaire (MLHFQ) }\end{array}$

\begin{tabular}{|c|c|c|c|}
\hline $\begin{array}{l}\text { Park, CL } \\
\text { y col}^{27}\end{array}$ & 2011 & EEUU & $\begin{array}{l}\text { Examinar la asociación entre } \\
\text { el sufrimiento religioso y el } \\
\text { bienestar mental y físico en } \\
\text { pacientes con ICA. }\end{array}$ \\
\hline
\end{tabular}

$\begin{array}{ll}\mathrm{N}=101 & - \text { Brief version of the Religious Strain Scale } \\ \text { pacientes } & - \text { Daily Spiritual Experiences Scale }\end{array}$

con ICA - SF-12 Health Survey.

- Satisfaction With Life Scale

- Minnesota Living with Heart Failure Questionnaire (MLHFQ)

- Center for Epidemiologic Studies Depression Scale (CES-D scale)

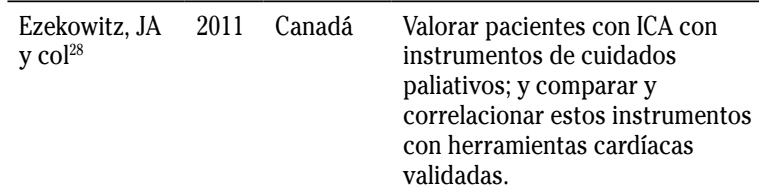

$\mathrm{N}=105 \quad-$ Edmonton Symptom Assessment Scale (ESAS)

pacientes - Kansas City Cardiomyopathy Questionnaire con ICA (KCCQ)

- Palliative Performance Scale (PPS)

- New York Heart Association (NYHA) functional class (I-IV).

- Minnesota Living with Heart Failure Questionnaire (MLHFQ)

\begin{tabular}{|c|c|c|c|}
\hline $\begin{array}{l}\text { Sela N } \\
\text { y col }^{29}\end{array}$ & 2011 & Israel & $\begin{array}{l}\text { Evaluar la influencia de Medical } \\
\text { Art therapy (MAT) en la calidad } \\
\text { de vida y el cumplimiento del } \\
\text { tratamiento médico en pacientes } \\
\text { con ICA }\end{array}$ \\
\hline $\begin{array}{l}\text { Huiskes, BL } \\
\mathrm{y} \mathrm{col}^{30}\end{array}$ & 2011 & EEUU & $\begin{array}{l}\text { Examinar múltiples factores } \\
\text { que tienen impacto potencial } \\
\text { en la calidad de vida de mujeres } \\
\text { con ICA y función sistólica } \\
\text { preservada. }\end{array}$ \\
\hline
\end{tabular}

\begin{tabular}{|c|c|c|c|}
\hline $\begin{array}{l}\text { Rogers, JG } \\
\mathrm{y} \mathrm{col}^{31}\end{array}$ & 2010 & EEUU & $\begin{array}{l}\text { Evaluar el impacto de los } \\
\text { dispositivos de asistencia } \\
\text { ventricular sobre la capacidad } \\
\text { funcional y la calidad de vida }\end{array}$ \\
\hline
\end{tabular}
$\mathrm{N}=22 \quad$ - Minnesota Living with Heart Failure pacientes Questionnaire [MLHFQ] con ICA

$\begin{array}{ll}\begin{array}{ll}\mathrm{N}=60 \\ \text { mujeres }\end{array} & - \text { Memorial Symptom Assessment Scale - HF } \\ \text { con ICA } & \text { - } \text { General Comfort Questionnaire [GCQ] } \\ & - \text { Minnesota Living with Heart Failure } \\ & \text { Questionnaire [MLHFQ] } \\ & - \text { 6-Minute Walk Test [6MWT] } \\ \mathrm{N}=281 & - \text { New York Heart Association (NYHA) functional } \\ \text { pacientes } & \text { class (I-IV). } \\ \text { con ICA } & - \text { 6-Minute Walk Distance (meters). } \\ & - \text { Minnesota Living with Heart Failure } \\ & \text { Questionnaire (MLHFQ) } \\ & - \text { Kansas City Cardiomyopathy Questionnaire } \\ & \text { (KCCQ) } \\ \text { N=433 } & - \text { Minnesota Living with Heart Failure } \\ \text { pacientes } & \text { Questionnaire [MLHFQ] } \\ \text { con ICA } & - \text { 6-Minute Walk Test [6MWT] } \\ & \end{array}$




\begin{tabular}{|c|c|c|c|}
\hline Autores & Año & País & Objetivo \\
\hline 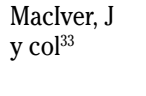 & 2008 & Canadá & $\begin{array}{l}\text { Determinar las preferencias de } \\
\text { tratamiento entre pacientes con } \\
\text { IC leve y ICA }\end{array}$ \\
\hline $\begin{array}{l}\text { Blinderman, } \\
\mathrm{CD} \mathrm{y} \mathrm{col}^{12}\end{array}$ & 2008 & EEUU & $\begin{array}{l}\text { Comprender las necesidades de } \\
\text { los pacientes con ICA acerca de } \\
\text { los síntomas y de otros factores } \\
\text { relacionados con la calidad de } \\
\text { vida. }\end{array}$ \\
\hline
\end{tabular}

\begin{tabular}{ll}
\multicolumn{1}{c}{ Población } & \multicolumn{1}{c}{ Instrumentos } \\
\hline N= 91 & - Minnesota Living with Heart Failure \\
pacientes & Questionnaire (MLHFQ) \\
(48 IC leve & - New York Heart Association functional class \\
y 43 con & (I-IV) (NYHA-FC) \\
ICA) & - Visual Analog Scale (VAS)
\end{tabular}

$\mathrm{N}=103$ con - Memorial Symptom Assessment Scale (MSAS)

IC grado - Mental Health Inventory-5 (MHI-5)

III/IV. - Multidimensional Index of Life Quality (MILQ)

- Functional Assessment of Chronic Illness Therapy-Spirituality Scale (FACIT-Spirituality)

- Short Portable Mental Status Questionnaire (SPMSQ) (Pfeiffer)

- Sickness Impact Profile (SIP)

\begin{tabular}{|c|c|c|c|c|}
\hline $\begin{array}{l}\text { Ter-Galstanyan, } \\
\text { A y coll }^{34}\end{array}$ & 2006 & EEUU & $\begin{array}{l}\text { Examinar la incidencia de } \\
\text { ansiedad y depresión en una } \\
\text { cohorte de pacientes con IC } \\
\text { sistólica avanzada }\end{array}$ & $\begin{array}{l}\mathrm{N}=241 \\
\text { pacientes } \\
(53,9 \% \text { con } \\
\text { ICA) }\end{array}$ \\
\hline $\begin{array}{l}\text { Hauptman, PJ } \\
\mathrm{y} \mathrm{col}^{35}\end{array}$ & 2006 & UK & $\begin{array}{l}\text { Determinar si el grado de } \\
\text { regurgitación mitral se } \\
\text { correlaciona con la calidad de } \\
\text { vida en pacientes que presentan } \\
\text { síntomas de IC. }\end{array}$ & $\begin{array}{l}\mathrm{N}=264 \\
\text { pacientes } \\
\text { (IC leve } \\
\text { y IC } \\
\text { moderada- } \\
\text { grave) }\end{array}$ \\
\hline $\begin{array}{l}\text { Sullivan, M } \\
\mathrm{y} \mathrm{col}^{36}\end{array}$ & 2004 & EEUU & $\begin{array}{l}\text { Estudiar la relación entre la } \\
\text { depresión y el estado de salud en } \\
\text { pacientes con ICA. }\end{array}$ & $\begin{array}{l}\mathrm{N}=113 \\
\text { pacientes } \\
\text { con ICA }\end{array}$ \\
\hline
\end{tabular}

- Brief Symptom Inventory-Anxiety

- The Patient Health Questionnaire Depression

Scale

- The Control Attitude Scale

$\begin{array}{ll}\mathrm{N}=264 & - \text { New York Heart Association functional class } \\ \text { pacientes } & \text { (I-IV) (NYHA-FC). } \\ \text { (IC leve } & - \text { 6-Minute Walk Distance (meters). } \\ \text { y IC } & - \text { Minnesota Living with Heart Failure } \\ \text { moderada- } & \text { Questionnaire (MLHFQ) } \\ \text { grave) } & - \text { SF-36 Health survey. }\end{array}$

$\mathrm{N}=113 \quad$ - Mini-Mental State Examination (MMSE)

pacientes - The Cumulative Illness Rating Scale-Geriatric con ICA (CIRS-G)

- Primary Care Evaluation of Mental Disorders (PRIME-MD)

- Hamilton Depression(HAM-D) Rating Scale

- The System Checklist 20 Depression Scale (SCL-20)

- The Hamilton Anxiety Rating Scale

\begin{tabular}{llll}
\hline $\begin{array}{l}\text { Hauptman, PJ 2004 } \\
\mathrm{y} \mathrm{col}^{37}\end{array}$ & EEUU & $\begin{array}{l}\text { Examinar la variabilidad del } \\
\text { estado de salud en pacientes con } \\
\text { ICA y compararlos con pacientes } \\
\text { con IC leve }\end{array}$
\end{tabular}

\begin{tabular}{|c|c|c|c|}
\hline $\begin{array}{l}\text { Evangelista, } \\
\text { LS y } \mathrm{col}^{22}\end{array}$ & 2003 & EEUU & $\begin{array}{l}\text { Describir y comparar los } \\
\text { comportamientos de los } \\
\text { pacientes de edad avanzada y los } \\
\text { pacientes más jóvenes con ICA en } \\
6 \text { actividades prescritas. }\end{array}$ \\
\hline
\end{tabular}

\begin{tabular}{lccl}
\hline Evangelista, & 2003 & EEUU & $\begin{array}{l}\text { Describir los efectos del } \\
\text { LS y col }{ }^{23}\end{array}$ \\
& & $\begin{array}{l}\text { tratamiento sobre los cambios } \\
\text { psicológicos en dos momentos de } \\
\text { su trayectoria de enfermedad }\end{array}$ \\
\hline
\end{tabular}

\begin{tabular}{lll}
\hline Jaarsma, T & 1996 & EEUU \\
$\mathrm{y} \mathrm{col}^{38}$ & &
\end{tabular}

Determinar el efecto de la baja

fracción de eyección en la función sexual en pacientes con ICA.
$\mathrm{N}=547$
pacientes
(118 con
ICA y 366
con IC leve-
moderada)
- New York Heart Association (NYHA) classification
- Kansas City Cardiomyopathy Questionnaire (KCCQ)
- The EQ-5D (formerly the EuroQOL)

$\mathrm{N}=140 \quad$ - HF Compliance Questionnaire pacientes con ICA
$\mathrm{N}=77$
pacientes
- The Beck Depression Inventory (BDI)
con ICA
- Physical Component Score (PCS)
- SF-12 Health Survey.

$\mathrm{N}=62$

pacientes

con ICA

- New York Heart Association functional class (I-IV) (NYHA-FC).

- 6-Minute Walk Distance (meters).

- The Psychosocial Adjustment to Illness Scale 


\section{VALORACIÓN DE SÍNTOMAS}

\section{Síntomas generales}

- Edmonton Symptom Assessment Scale (ESAS)

- The Memorial Symptom Assessment Scale HF(MSAS-HF)

- The Cumulative Illness Rating Scale-Geriatric (CIRS-G)

- Visual Analog Scale (VAS)

\section{VALORACIÓN PSICOLÓGICA Y COGNITIVA}

- Hospital Anxiety and Depression Scale (HADS)

- Hamilton Depression Rating Scale (HAM-D)

- The System Checklist 20 Depression Scale (SCL-20)

- The Patient Health Questionnaire Depression Scale

- Beck Depression Inventory II (BDI-II)

- Center for Epidemiologic Studies Depression Scale (CES-D scale)

- The Hamilton Anxiety Rating Scale (HAM-A)

- Brief Symptom Inventory-Anxiety

- Mini-Mental State Examination (MMSE)

- Short Portable Mental Status Questionnaire (SPMSQ)

- Mini-Cog Test

- Montreal Cognitive Assessment

- Primary Care Evaluation of Mental Disorders (PRIME-MD)

- The Rand Mental Health Inventory (MHI)

- Mental Health Inventory-5 (MHI-5)

- Brief Symptom Inventory (BSI)

- Palliative Performance Scale (PPS)

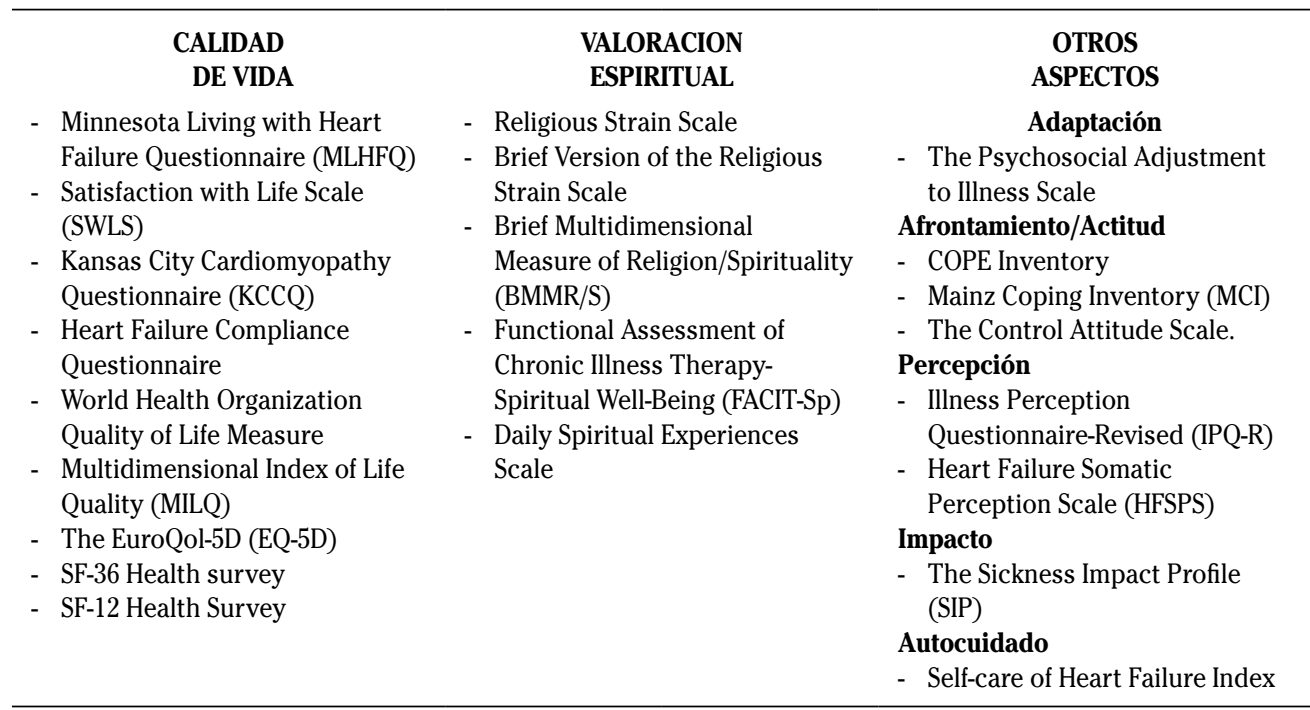

Figura 2. Instrumentos utilizados en la valoración del paciente con ICA.

Para este trabajo se ha considerado instrumento a cualquier tipo de cuestionario, escala, test o prueba funcional, que se utilice para valorar algún aspecto de la persona con ICA. El proceso de extracción de estas herramientas en cada artículo se realizó en dos fases. En una primera fase, se extrajeron los instrumentos utilizados para valorar al paciente con ICA de cada uno de los artículos seleccionados. Poste- riormente, en la segunda fase, y de forma consensuada por dos de los investigadores, se decidió realizar un esquema de los instrumentos obtenidos dividiéndolos por esferas de valoración del paciente con ICA; facilitando de esta manera la exposición y comprensión de las herramientas halladas. Finalmente, se identificaron aquellos instrumentos que fueron más utilizados, recopilando información sobre ellos en 
referencia a: la población para la cual fue diseñado, validación, traducción al castellano y aplicabilidad en la práctica clínica.

\section{RESULTADOS}

A través de los estudios seleccionados se encontraron un total de 49 instrumentos, divididos por esferas de valoración en el paciente con ICA, tal y como se muestra en la figura 2. Todos los artículos seleccionados corresponden a estudios cuantitativos excepto uno, que se trata de una revisión sobre la valoración del deterioro cognitivo en pacientes con ICA. Igualmente, se ha encontrado que la mayoría de los mismos han sido publicados entre los años $2010 \mathrm{y}$ 2014 por lo que la evidencia hallada es muy reciente. Los temas más relevantes tratados en los estudios han sido la descripción y valoración de los síntomas físicos, psicológicos, espirituales y de calidad de vida; y cómo influye la relación entre ellos en el paciente con ICA.

Los instrumentos más utilizados en cada una de las esferas de valoración del paciente con ICA han sido: valoración de síntomas, valoración psicológica y cognitiva, valoración de calidad de vida y valoración espiritual.

\section{Valoración de síntomas}

Los instrumentos incluidos en la esfera de valoración de síntomas han sido dividido a su vez en tres grupos: síntomas generales, síntomas específicos y valoración funcional.

\section{Síntomas generales}

En lo que respecta a los síntomas generales, se han encontrado cuatro instrumentos: Edmonton Symptom Assessment Scale (ESAS), The Memorial Symptom Assessment Scale - Heart Failure (MSASHF), The Cumulative Illness Rating ScaleGeriatric (CIRS-G) y Visual Analog Scale (VAS). Sin embargo, predomina el uso de dos instrumentos para la valoración de los síntomas generales: el ESAS y el MSASHF. El primero de ellos, fue diseñado para hacer frente a los síntomas complejos que experimentaban los pacientes con cáncer avanzado, permitiendo realizar mediciones cuantitativas de los nueve síntomas más comunes que padecen estos pacientes. Este instrumento se encuentra validado y traducido al español, y ha sido ampliamente utilizado en la práctica clínica, y también en pacientes con ICA, tanto en el medio hospitalario como en la atención domiciliaria, resultando apropiado su uso clínico por su sencillez ${ }^{39}$.

Por otro lado, el MSAS-HF surgió como una modificación del Memorial Sympton Assessment Scale para medir los síntomas específicos de pacientes con IC. con una validez y fiabilidad aceptables ${ }^{40}$, su desventaja frente el ESAS es el mayor número de ítems, 32 frente a 9. Actualmente, no se han encontrado estudios acerca de la validación y traducción al castellano del mismo.

\section{Sintomas específicos}

Se disponen para la valoración de síntomas específicos de las siguientes herramientas: The Short Form of the McGill Pain Questionnaire (SF-MPQ), The Brief Pain Inventory (BPI), Epworth Sleepiness Scale (ESS) y Mini Nutritional Assessment (MNA). Destacan por su frecuente utilización el BPI y el SF-MPQ para la valoración del dolor. En relación con este último, el SF-MPQ fue diseñado para describir las dimensiones sensitivas y afectivas del dolor en pacientes con dolor crónico ${ }^{41}$. Además, con respecto al McGill Pain Questionnaire, requiere menos tiempo de una hora para rellenarlo, lo que le hace útil en la práctica clínica. Sin embargo, la versión reducida (SF-MPQ) no cuenta con estudios acerca de su validación y traducción al castellano.

Por otra parte, en lo que se refiere al BPI, fue diseñado para pacientes con dolor a causa de enfermedades o condiciones crónicas como el cáncer y la osteoartritis, así como para el dolor agudo, como por ejemplo en casos de dolor postoperatorio. Tanto la versión original como las versiones levemente modificadas, muestran una excelente validez y fiabilidad ${ }^{42}$. Igualmente, ha sido utilizado ampliamente para valorar 
el dolor en distintas enfermedades y cáncer, encontrándose validado y traducido al castellano $^{43}$.

\section{Valoración funcional}

Para medir la valoración funcional se han hallado 3 instrumentos: New York Heart Association Functional Class (NYHAFC), 6 Minute Walk Distance (6MWT) y Palliative Performance Scale (PPS). En las investigaciones prima la utilización de la NYHA Functional Class y el 6-Minute Walk Distance para la valoración del estado funcional de estos pacientes. Comúnmente utilizada como método para la clasificación funcional de pacientes con insuficiencia cardíaca, la NYHA-FC designa 4 clases (I, II, III y IV) basándose en las limitaciones en la actividad física del paciente ocasionadas por los síntomas cardíacos. Así mismo, tiene un importante valor pronóstico y se utiliza como criterio decisivo en la elección de determinadas intervenciones terapéuticas, tanto médicas como quirúrgicas ${ }^{44}$. Por otra parte, el 6MWT se puede utilizar para medir el estado funcional de pacientes cardíacos y pulmonares, así como para fines epidemiológicos. Sin embargo, su principal limitación en la práctica clínica es que la prueba debe ser realizada bajo la supervisión de personal cualificado de acuerdo con el protocolo estándar descrito por la American Thoracic Society ${ }^{45}$.

\section{Valoración psicológica y cognitiva}

\section{Valoración psicológica}

Existen una amplia variedad de instrumentos utilizados en la valoración psicológica de estos pacientes ( $n=8$ ) (Fig. 3). Todos ellos muestran una validez, fiabilidad y sensibilidad comprobadas. Sin embargo, el uso del Hospital Anxiety and Depression Scale (HADS) predomina en comparación con el resto. Este instrumento fue diseñado para identificar y cuantificar la ansiedad y la depresión de pacientes en consultas ambulatorias $^{46}$. Investigaciones realizadas en España, y en más de 200 estudios publicados de todo el mundo, han informado de resultados clínicamente significativos del HADS como herramienta de evaluación psicológica, comprobándose ampliamente su validez y fiabilidad. Además, es comúnmente utilizado en la práctica diaria ya que demuestra ser sensible a los cambios, tanto durante el transcurso de las enfermedades como en la respuesta a intervenciones psicoterapéuticas y psicofarmacológicas ${ }^{47}$. Así mismo, se encuentra validado y traducido al español ${ }^{48}$.

\section{Valoración cognitiva}

En la actualidad se disponen de un gran número de herramientas en lo que respecta a la valoración cognitiva. Entre los instrumentos más empleados destacan el Mini Mental State Examination (MMSE) y el Short Portable Mental Status Questionnaire (SPM$\mathrm{SQ})$. El primero de ellos fue diseñado con la idea de proporcionar un análisis breve y estandarizado del estado mental que sirviera para diferenciar, en pacientes psiquiátri$\cos$, los trastornos funcionales orgánicos ${ }^{47}$. Existen numerosos estudios de validación y fiabilidad, desarrollándose una versión adaptada y estandarizada de este instrumento en la población española ${ }^{49}$. Debido a su sencillez y brevedad constituye una de las pruebas más usadas tanto en la clínica como en la investigación.

Por otro lado, el SPMSQ desarrollado para la evaluación de la función cognitiva en personas mayores, se encuentra entre los cuestionarios de cribado más recomendados. Validado y traducido al castellano, su utilidad estriba en que es una prueba fácil de realizar, sin requerimiento de material específico para su cumplimentación, siendo aplicable a personas de bajo nivel de escolarización ${ }^{50}$.

\section{Valoración de la calidad de vida}

Entre los 9 instrumentos identificados en la literatura para la valoración de la calidad de vida en pacientes cardíacos avanzados, predomina la utilización del Minnesota Living with Heart Failure questionnaire (MLHFQ) y el Kansas City Cardiomyopathy Questionnaire (KCCQ). En lo 
que respecta al MLHFQ, fue diseñado para medir la percepción de los efectos de la IC en los aspectos físicos, socioeconómicos y psicológicos de la vida del paciente en el último mes. Es ampliamente utilizado y particularmente útil en la población con ICA porque es corto, fácil de entender por los enfermos y ancianos, auto-administrado, y fácil de puntuar ${ }^{32,33}$. Es el instrumento más utilizado para la evaluación de la calidad de vida en pacientes con IC, y ha sido traducido y validado al español, y a más de 32 lenguas, demostrando buenas propiedades psicométricas en numerosos estudios ${ }^{51}$.

En cambio, el KCCQ es el instrumento más reciente y el único que, además de valorar las dimensiones clásicas de los cuestionarios de calidad de vida (física, síntomas, social), incorpora una valoración de los cambios en los síntomas y en el nivel de autocuidado. Validada y traducida al castellano, ha demostrado ser una herramienta fiable y sensible a los cambios clínicos ${ }^{19,37}$. Por lo que su selección en la práctica clínica y la investigación como instrumento para monitorizar la calidad de vida de estos pacientes se debe tener en consideración $^{52}$.

\section{Valoración espiritual}

Las herramientas halladas en lo que respecta a la valoración espiritual son las siguientes: Religious Strain Scale y su versión corta, Daily Spititual Experiences Scale, Brief Multidimensional Measure of Religion/Spirituality (BMMR/S) y Functional Assessment of Chronic Illness Therapy-Spiritual Wellbeing (FACIT-Sp). Sin embargo, escasas investigaciones se han desarrollado en este aspecto en el paciente con ICA, salvo las realizadas por Park y col $^{15,27}$ y Strada y $\mathrm{col}^{20}$. Por ello, no predomina el empleo de algún instrumento por encima de otro. Se conoce que todas ellas muestran unas propiedades de validez y fiabilidad adecuadas ofreciendo opciones para su uso en la práctica clínica ${ }^{15,20,27}$. No obstante, únicamente se encuentran validados y traducidos al español, el Daily Spiritual Experiences Scale y el FACIT-Sp ${ }^{53}$.

\section{Otros aspectos}

En esta sección se han incluido una serie de herramientas que evalúan diferentes aspectos del paciente con ICA, como la adaptación, el afrontamiento y la percepción de la enfermedad entre otros. Sin embargo, no se han desarrollado debido a que el empleo de estos instrumentos en los artículos ha sido escaso, por lo que no han destacado como los vistos en las esferas de valoración comentadas anteriormente.

\section{DISCUSIÓN}

La revisión sistemática de la literatura identificó 49 instrumentos que han sido utilizados para valorar al paciente con ICA. Entre los mismos, 7 (14\%) fueron específicamente diseñados para pacientes cardíacos y se ha demostrado su utilidad en la fase avanzada de IC. Dos de los instrumentos (4\%) fueron diseñados para el paciente oncológico avanzado, como son el caso del ESAS y el PPS. Sin embargo, a través de su utilización en los estudios se conoce que proporcionan datos fiables y válidos en pacientes con ICA, por lo que son utilizados para su valoración tanto en el ámbito hospitalario como domiciliario. Finalmente, el resto de herramientas halladas (85\%) son genéricas y han demostrado ser útiles para esta población.

Los pacientes con ICA presentan diferentes síntomas comunes y angustiantes durante los últimos 6 meses de vida ${ }^{12}$, quizás por ello, el número de instrumentos encontrados en el área de valoración de síntomas es alto $(\mathrm{n}=11)$. En la mayoría de los estudios el objetivo de la valoración sintomática del paciente era conocer el estado general del mismo, en función de los síntomas generales y la valoración funcional, y no en torno a problemas o síntomas específicos. Por ello, el empleo de instrumentos para valorar síntomas específicos ha sido escaso, siendo únicamente utilizados en 3 de los 27 artículos seleccionados.

No parece necesario el diseño de nuevos instrumentos que se centren en la valoración sintomática debido al número de instrumentos encontrados. Sin embargo, 
se abren opciones de futuras investigaciones en lo que se refiere a adaptación cultural y validación al castellano, ya que no se disponen de estas versiones en el MSAS-HF y SF-MPQ. Por ello, se propone que se tengan en cuenta la brevedad y el formato propicio en el caso de la adaptación de estos instrumentos para la práctica clínica.

Por otro lado, existen una amplia variedad de instrumentos utilizados en la valoración psicológica y cognitiva $(n=16)$. Esto puede deberse a que síntomas como la presencia de disnea, fatiga, falta de capacidad de ejercicio y dificultades para dormir a menudo conducen a la depresión, la ansiedad y la baja autoestima. De hecho, se estima que entre el 25 y el $50 \%$ de los pacientes con insuficiencia cardíaca presenta ansiedad, y del 18 al 47\% depresión, todo ello dependiendo de la edad y duración del diagnóstico ${ }^{25}$. En lo que se refiere a la valoración cognitiva, se disponen de un gran número de herramientas, tal vez debido a que estos pacientes están en mayor riesgo de deterioro cognitivo, particularmente en la función ejecutiva y de memoria ${ }^{22}$. En realidad, la utilización de instrumentos como el MMSE o el SPMSQ es importante, debido al riesgo potencial de hospitalizaciones, incumplimiento del tratamiento y afectación en la toma de decisiones que presentan estos pacientes ${ }^{24}$. Estas evaluaciones realizadas de forma frecuente pueden ser útiles para anticipar problemas y evaluar la respuesta al tratamiento. Igualmente, se debe considerar una evaluación cognitiva más amplia en pacientes que experimenten descensos significativos en habilidades de referencia ${ }^{24}$.

Así mismo, se conoce que la ICA deteriora la calidad de vida de los pacientes en varias dimensiones. Algunos comentan las alteraciones que se han producido en sus vidas cotidianas, debido a que han perdido la capacidad de participar en sus pasatiempos favoritos, actividades y otros asuntos. Así mismo, explican cómo su enfermedad da lugar a la soledad, la pérdida de amigos y a una vida social limitada. La lucha contra este aislamiento constituye un problema constante para estas personas, no solo debido a las limitaciones físicas, sino debido al sentimiento de abandono por sus familiares y amigos. Junto a esto, a su vez, se sienten incapaces de planificar el futuro por no saber lo que les deparará el día a día debido a los síntomas ${ }^{54}$. De esta manera, la calidad de vida de los pacientes con ICA se puede ver comprometida, provocando que incluso, en ocasiones, deseen su muerte ${ }^{55}$. En este sentido, algunos investigadores han encontrado peores resultados clínicos, en términos de mortalidad y reingresos hospitalarios, en pacientes con disminución en la calidad de vida ${ }^{32}$. Dado lo anterior, no es de extrañar que la valoración de la misma en pacientes cardíacos avanzados constituya un aspecto fundamental en numerosos estudios, encontrándose 9 instrumentos en esta parcela de valoración.

Finalmente, los temas de la espiritualidad son importantes para muchos de los pacientes con IC, y se vuelven especialmente relevantes cuando su enfermedad avanza y empeora. Varios estudios transversales han demostrado que los aspectos positivos que aportan la espiritualidad y religiosidad son relacionados con un mejor bienestar psicológico de las personas que viven con $\mathrm{IC}^{27}$. A pesar de encontrar 5 instrumentos para su valoración, como ya se ha comentado, únicamente se encuentran traducidos al español el Daily Spiritual Experiences Scale y el FACIT-Sp, por consiguiente se abren opciones de futuras investigaciones en lo que se refiere a adaptación cultural y validación al castellano de varios instrumentos. Además, el área de valoración espiritual constituye un amplio escenario para desarrollar investigaciones ya que escasos estudios se han desarrollado en este ámbito en el paciente con ICA.

A través de esta revisión, se alienta a los profesionales de la salud a utilizar algunos de los instrumentos hallados en su práctica clínica diaria con estos pacientes. El hecho de emplear estas herramientas en la práctica sanitaria profesional ofrece opciones a dichos profesionales para valorar mejor las necesidades específicas de esta población y prevenir posibles complicaciones, lo que incrementaría la calidad de los cuidados que los pacientes reciben y por lo 
tanto, su calidad de vida. De esta manera, a su vez, será posible planificar intervenciones, así como valorar la eficacia de las mismas, ayudando a las personas con ICA a encontrar nuevas formas y alternativas de lidiar con las restricciones, desafíos y oportunidades que enfrentan en su vida cotidiana. Y por lo tanto, garantizar que las necesidades de estos enfermos se satisfagan adecuadamente.

Otro aspecto importante a destacar, es que cuando los profesionales estén seleccionando instrumentos para valorar a pacientes con ICA, deben identificar si son fáciles de utilizar en la práctica clínica diaria, ya que no sólo necesitan instrumentos con buenas propiedades psicométricas, sino que también requieren de herramientas relativamente breves y sencillas de administrar.

Finalmente, con respecto a las limitaciones de la revisión, puede que no se hayan identificado algunas publicaciones debido a la restricción de idioma (inglés o español). Igualmente, también hay que tener en cuenta que solamente se han desarrollado los instrumentos más utilizados en la valoración del paciente con ICA.

Por otro lado, como puntos fuertes de la misma, se pueden destacar tanto la rigurosa selección de artículos, a través de unos criterios de inclusión y exclusión específicos y definidos, como el análisis de contenido exhaustivo en cada uno de ellos. Al mismo tiempo, que la verificación de la selección de los estudios haya sido realizada por varios investigadores confiere calidad y rigor al estudio. Por último, la información que se proporciona sobre los instrumentos más utilizados, como la traducción y validación en castellano, y su aplicabilidad en la práctica clínica, es especialmente útil para los profesionales de nuestro país.

En conclusión, la valoración de los pacientes con ICA es un tema en auge al considerar el aumento de artículos al respecto en los últimos años.

Los profesionales de la salud tienen acceso a instrumentos válidos y fiables para valorar a estas personas en todas sus dimensiones. Este estudio demuestra la gran variedad de herramientas eficaces y aplicables para valorar las diferentes esferas de la persona con ICA. Su integración en la práctica clínica diaria ofrece opciones a los profesionales sanitarios en la valoración e identificación de necesidades específicas, lo que permitiría mejorar el control de síntomas y el manejo de complicaciones. Por otra parte, la adaptación y la validación al castellano de algunos instrumentos sería de gran utilidad, como es en el caso de los instrumentos para la valoración espiritual.

\section{BIBLIOGRAFÍA}

1. Banegas JR, Villar F, Graciani A, Rodríguez-ArTALEJO F. Epidemiología de las enfermedades cardiovasculares en España. Rev Esp Cardiol 2006; 6: 3-12.

2. Sayago-Silva I, García-López F, Segovia-Cubero J. Epidemiology of heart failure in Spain over the last 20 years. Rev Esp Cardiol 2013; 66: 649-656.

3. González-Juanatey JR, Alegría Ezquerra E, Bertoméu Martínez V, Conthe Gutiérrez P, De SaNtiago Nocito A, Zsolt Fradera I. Insuficiencia cardiaca en consultas ambulatorias: comorbilidades y actuaciones diagnóstico-terapéuticas por diferentes especialistas. Estudio EPISERVE. Rev Esp Cardiol 2008; 61: 611-619.

4. Instituto Nacional de Estadística. Defunciones según la causa de muerte 2013. 2013; Accedido el 05/06/2015, disponible en: http:// www.ine.es

5. Muñoz Bautista AM, Cruz Díaz R, Vélez Pérez Y, Puig Calvet M, Durán Escuadra CL, Vila Mir M, et al. Caso clínico: Continuidad asistencial en un paciente con insuficiencia cardiaca avanzada en tratamiento con Dobutamina. Enferm Cardiol 2011; 53-54: 82-88.

6. Goodlin SJ, Hauptman PJ, Arnold R, Grady K, Hershberger RE, Kutner J, et al. Consensus statement: palliative and supportive care in advanced heart failure. J Card Fail 2004; 10 : 200-209.

7. Deng MC, NAKA Y. Mechanical Circulatory Support Therapy in Advanced Heart Failure. London: Imperial College Press; 2007.

8. Hernandez AF, Felker GM. Advanced Heart Failure. Prog Cardiovasc Dis 2011; 54: 77.

9. Formiga F, Manito N, Pujol R. Revisión: Insuficiencia cardíaca terminal. Med Clin 2007; 128: 263-267. 
10. LóPEz CASTRO J. Insuficiencia cardíaca: un enfoque multidisciplinar. España: ECU; 2013.

11. Lemond L, Allen LA. Palliative Care and Hospice in Advanced Heart Failure. Prog Cardiovasc Dis 2011; 54: 168-178.

12. Blinderman CD, Homel P, Billings JA, Portenoy RK, Tennstedt SL. Symptom distress and quality of life in patients with advanced congestive heart failure. J Pain Symptom Manage 2008; 35: 594-603.

13. Hauptman PJ, Masoudi FA, Weintraub WS, Pina I, JoNEs PG, SPERTUS JA. Variability in the clinical status of patients with advanced heart failure. J Card Fail 2004; 10: 397-402.

14. Evangelista LS, Liao S, Motie M, De Michelis N, Ballard-Hernandez J, Lombardo D. Does the type and frequency of palliative care services received by patients with advanced heart failure impact symptom burden? J Palliat Med 2014; 17: 75-79.

15. Park CL, Lim H, Newlon M, Suresh DP, Bliss DE. Dimensions of religiousness and spirituality as predictors of well-being in advanced chronic heart failure patients. J Relig Health 2014; 53: 579-590.

16. Bunyamin V, Spaderna H, Weidner G. Health behaviors contribute to quality of life in patients with advanced heart failure independent of psychological and medical patient characteristics. Qual Life Res 2013; 22: 1603-1611.

17. Aggarwal a, Kumar A, Gregory mp, Blair C, PaumaA S, Tatooles A, J., et al. Nutrition Assessment in Advanced Heart Failure Patients Evaluated for Ventricular Assist Devices or Cardiac Transplantation. Nutr Clin Pract 2013; 28: 112-119.

18. Lennie TA, Moser DK, Biddle MJ, Welsh D, BrucKNER GG, ThOMAs DT, et al. Nutrition intervention to decrease symptoms in patients with advanced heart failure. Res Nurs Health $2013 ; 36: 120-145$.

19. Goodlin SJ, Wingate S, Albert NM, Pressler SJ, Houser J, Kwon J, et al. Investigating Pain in Heart Failure Patients: The Pain Assessment, Incidence, and Nature in Heart Failure (PAINHF) Study. J Card Fail 2012;18: 776-783.

20. Strada EA, Homel P, Tennstedt S, Billings JA, Portenoy RK. Spiritual well-being in patients with advanced heart and lung disease. Palliat Support Care 2013; 11: 205-213.

21. Buck HG, Lee CS, Moser DK, Albert NM, Lennie T, BENTLEY B, et al. Relationship between selfcare and health-related quality of life in older adults with moderate to advanced heart failure. J Cardiovasc Nurs 2012; 27: 8-15.

22. Evangelista LS, Doering LV, Dracup K, Westlake C, Hamilton M, Fonarow GC. Compliance beha- viors of elderly patients with advanced heart failure. J Cardiovasc Nurs 2003; 18: 197-208.

23. Evangelista LS, Westlake C, Dracup K, Erickson $\mathrm{V}$, Fonarow GC. Do psychological scores improve as an effect of time and treatment in advanced heart failure patients? J Card Fail 2003; 9: S78.

24. Gaviria M, Pliskin N, Kney A. Cognitive impairment in patients with advanced heart failure and its implications on decision-making capacity. Congest Heart Fail 2011; 17(4): 175-179.

25. Hallas CN, Wray J, Andreou P, Banner NR. Depression and perceptions about heart failure predict quality of life in patients with advanced heart failure. Heart Lung 2011; 40: 111-121.

26. Lee CS, Kim AY, Green J, Rosenfeld A. Symptom Profiles in Advanced Heart Failure with Marked Differences in Quality-of-Life. J Card Fail 2011; 17: S76.

27. Park CL, Wortmann JH, Edmondson D. Religious struggle as a predictor of subsequent mental and physical well-being in advanced heart failure patients. J Behav Med 2011; 34: 426436.

28. Ezekowitz JA, Thai V, Hodnefield TS, SAnderson L, CUJEC B. The correlation of standard heart failure assessment and palliative care questionnaires in a multidisciplinary heart failure clinic. J Pain Symptom Manage 2011; 42: 379387.

29. Sela N, Baruch N, Assali A, Vaturi M, Battler A, BEN GAL T. The influence of medical art therapy on quality of life and compliance of medical treatment of patients with advanced heart failure. Harefuah 2011; 150: 79-83, 209.

30. Huiskes BL, Heywood JT, Dracup K. Comfort and Symptom Burden Associated with Health-Related Quality of Life in Older Women with Advanced Heart Failure and Preserved Systolic Function. J Card Fail 2011; 17: S5.

31. Rogers JG, Aaronson KD, Boyle AJ, Russell SD, Milano CA, Pagani FD, et al. Continuous Flow Left Ventricular Assist Device Improves Functional Capacity and Quality of Life of Advanced Heart Failure Patients. J Am Coll Cardiol 2010; 55: 1826-1834.

32. Moser DK, Yamokoski L, Sun JL, Conway GA, Hartman KA, Graziano JA, et al. Improvement in health-related quality of life after hospitalization predicts event-free survival in patients with advanced heart failure. J Card Fail 2009; 15: 763-769.

33. Maciver J, Rao V, Delgado DH, Desai N, Ivanov J, AbBey S, et al. Choices: a Study of Preferences for End-of-life Treatments in Patients With Advanced Heart Failure. J Heart Lung Transplant 2008; 27: 1002-1007. 
34. Ter-Galstanyan A, Evangelista LS, Moser DK, Lennie T, Dejong M, Chung MS, et al. What factors increase anxiety and depression in advanced heart failure? J Card Fail 2006; 12 : S108.

35. Hauptman PJ, Rector TS, Wentworth D, Kubo S. Quality of life in advanced heart failure: Role of mitral regurgitation. Am Heart J 2006; 151: 213-218.

36. Sullivan M, Levy WC, Russo JE, Spertus JA. Depression and health status in patients with advanced heart failure: a prospective study in tertiary care. J Card Fail 2004; 10: 390-396.

37. Hauptman PJ, Masoudi FA, Weintraub WS, Pina I, JoNEs PG, SPERTUS JA. Variability in the clinical status of patients with advanced heart failure. J Card Fail 2004; 10: 397-402.

38. JaArsma T, Dracup K, Walden J, Stevenson Lw. Sexual function in patients with advanced heart failure. Heart Lung 1996; 25: 262-270.

39. Carvajal A, Hribernik N, Duarte E, Sanz-Rubiales A, Centeno C. The Spanish Version of the Edmonton Symptom Assessment System-Revised (ESAS-r): First Psychometric Analysis Involving Patients With Advanced Cancer. J Pain Symptom Manage 2013; 45:129-136.

40. Zambroski CH, Lennie T, Chung ML, Heo S, Smoot T, ZIEGLER C. Use of the memorial symptom assessment scale-heart failure in heart failure patients. Circulation 2004; 110: 739-739.

41. Dudgeon D, Raubertas RF, Rosenthal SN. The short-form McGill Pain Questionnaire in chronic cancer pain. J Pain Symptom Manage 1993; 8: 191-195.

42. FURLER L. Validity and reliability of the pain questionnaire "Brief Pain Inventory". A literature research. Pflege Z 2013 Sep; 66: 546550.

43. Badia X, Muriel C, Gracia A, Manuel Núñez-Olarte J, Perulero N, Gálvez R, et al. Validación española del cuestionario Brief Pain Inventory en pacientes con dolor de causa neoplásica. Med Clin 2003; 120: 52-59.

44. Rostagno C, Galanti G, Comeglio M, Boddi V, Olivo G, Serneri G. Comparison of different methods of functional evaluation in patients with chronic heart failure. Eur J Heart fail 2000; 2 : 273-280.

45. ATS Committee on Proficiency Standards for Clinical Pulmonary Function Laboratories.
ATS statement: guidelines for the six-minute walk test. Am J Respir Crit Care Med 2002; 166: 111-117.

46. QuinTANA JMP. Evaluation of the psychometric characteristics of the Spanish version of the Hospital Anxiety and Depression Scale. Acta Psychiatr Scand 2003; 107: 216-221.

47. Herrmann C. International experiences with the hospital anxiety and depression scale: A review of validation data and clinical results. J Psychosom Res 1997; 42: 17-41.

48. Herrero Mu, Blanch J, Peri Ju, De Pablo J, Pintor L, BulbenA A. A validation study of the hospital anxiety and depression scale (HADS) in a Spanish population. Gen Hosp Psychiatry 2003; 25: 277-283.

49. Mitchell AJ, Shukla D, Ajumal HA, Stubbs B, TAHIR TA. The Mini-Mental State Examination as a diagnostic and screening test for delirium: systematic review and meta-analysis. Gen Hosp Psychiatry 2014; 36: 627-633.

50. Martínez De la Iglesia J, Dueñas Herrero R, Carmen Onís Vilchesa M, Aguado Tabernéa C, Albert Colomerc C, LuQue LuQuec R. Adaptación y validación al castellano del cuestionario de Pfeiffer (SPMSQ) para detectar la existencia de deterioro cognitivo en personas mayores de 65 años. Med Clin 2001; 117: 129-134.

51. Garin O, Soriano N, Ribera A, Ferrer M, Pont À, Alonso J, et al. Validación de la versión española del Minnesota Living with Heart Failure Questionnaire. Rev Esp Cardiol 2008; 61: 251259.

52. Comín-Colet J, Garin O, Lupón J, Manito N, CresPo-Leiro M, Gómez-Bueno M, et al. Original article: Validation of the Spanish Version of the Kansas City Cardiomyopathy Questionnaire. Rev Esp Cardiol (English Edition) 2011; 64: 51-58.

53. Questionnaires. Disponible en: http://www. facit.org/FACITOrg/Questionnaires. Accedido 15/02/2015, 2015.

54. Hopp FP, Thornton N, Martin L. The Lived Experience of Heart Failure at the End of Life: A Systematic Literature Review. Health Soc Work 2010; 35: 109-117.

55. Boyd KJ, Murray SA, Kendall M, Worth A, FreDERICK Benton T, Clausen H. Living with advanced heart failure: a prospective, community based study of patients and their carers. Eur J Heart Fail 2004; 6: 585-591. 